\title{
Metabólitos especiais isolados de Laseguea erecta (Apocynaceae)
}

\author{
Mario G. de Carvalho ${ }^{1 *}$, Cássia C.F. Alves ${ }^{1,2}$, Alceni A. Werle ${ }^{3}$, Raimundo Braz-Filho $^{4}$ \\ ${ }^{1}$ Departamento de Química, ICE, Uiversidade Federal Rural do Rio de Janeiro, \\ 23851-970, Seropédica, RJ, Brasil, \\ ${ }^{2}$ Depatamento de Química, Campus de Arraias, Universidade Federal do Tocantins, \\ 77330-000, Arraias, TO, Brasil, \\ ${ }^{3}$ Departamento de Química, ICEB, Universidade Federal de Ouro Preto, 35400-000, \\ Ouro Preto, MG, Brasil, \\ ${ }^{4}$ Setor de Química de Produtos Naturais, Universidade Estadual Norte Fluminense, \\ 28013-603, Campos, RJ, Brasil
}

\begin{abstract}
RESUMO: Do caule de Laseguea erecta foram isolados o lupeol, a cumarina escopoletina, o lignóide pinoresinol e o glicosil-cardenoídeo, $\alpha$-L-tevetosil-digitoxigenina. As estruturas foram identificadas com o uso de técnicas espectroscópicas de IV, $\mathrm{RMN}{ }^{1} \mathrm{H} \mathrm{e}{ }^{13} \mathrm{C}$, incluindo técnicas especiais $1 \mathrm{D}$ e $2 \mathrm{D}$, espectroscopia de massas e comparação com dados da literatura.
\end{abstract}

Unitermos: Laseguea erecta, Apocynaceae, glicosil-cardenoídeo, escopoletina, lignana.

\begin{abstract}
Special metabolites isolated from Laseguea erecta (Apocynaceae)". From the stem of Laseguea erecta the triterpene lupeol, the cumarine scopoletin, the lignoid pinoresinol and digitoxigenin $\alpha$-L-tevetoside, known as thevetioside, were isolated. The structures were identified by IR, mass, ${ }^{1} \mathrm{H}$ and ${ }^{13} \mathrm{C}$ NMR spectroscopy, including 1D and 2D techniques, together with comparison with literature data.
\end{abstract}

Keywords: Laseguea erecta, Apocynaceae, cardenolide glycoside, scopoletin, lignan.

\section{INTRODUÇÃO}

Espécies de Apocynaceae são conhecidas como bioprodutoras de alcalóides, terpenóides e iridóides (Carvalhaes et al., 2002; Vanderlei et al., 1991; Barreto et al., 1998), ocorrendo com menor frequência os flavonóides glicosilados (Carvalho et al., 2001a). O estudo fitoquímico de espécies representantes dos gêneros desta família, e cuja química ainda é pouco conhecida, pode conduzir à descoberta de novas fontes de substâncias naturais ativas uma vez que são frequentemente citadas em trabalhos de revisão sobre plantas com atividades farmacológicas e medicinais (Barbosa-Filho et al., 2006; Falcão et al., 2005; Morais et al., 2005). Este é o primeiro trabalho relacionado a estudo fitoquímico de uma espécie do gênero Laseguea e revela-o como bioprodutor de diferentes classes de metabólitos especiais, como lignóides, cumarinas e glicosídeos cardiotônicos. A presença dessas classes de compostos neste gênero revela o seu potencial para ser investigado através de estudos farmacognósticos. Isto se caracteriza não só devido às atividades dos arilpropanóides, como antibacterial das cumarinas e inibição da AMP fosfodiesterase cíclica do pinoresinol e derivados (Nishibe, 1989) mas, também, pela presença da digitoxina. A presença deste heterosídeo reforça a atenção a este gênero nesta área de conhecimento, uma vez que é um representante dos heterosídios esterólicos que são estimulantes e tônicos do coração (Souza et al., 2004).

\section{MATERIAL E MÉTODOS}

\section{Equipamentos}

Os dados espectrométricos no infravermelho foram obtidos em aparelho Perkin-Elmer FT-IR1600/1605 usando pastilha de $\mathrm{KBr}$ ou filme de $\mathrm{NaCl}$ e as freqüências de absorção foram medidas $\mathrm{em} \mathrm{cm}^{-1}$. Os espectros de RMN foram obtidos em espectrômetro Bruker $200 \mathrm{MHz}\left({ }^{1} \mathrm{H}\right)$ e $50 \mathrm{MHz}\left({ }^{13} \mathrm{C}\right)$ e Jeol JNM-GX$400400 \mathrm{MHz}\left({ }^{1} \mathrm{H}\right)$ e $100 \mathrm{MHz}\left({ }^{13} \mathrm{C}\right)$, usando $\mathrm{CDCl}_{3}$ e $\mathrm{D}_{3} \mathrm{CCOCD}_{3}$ como solventes e tetrametilsilano (TMS) com referência interna. Os espectros de massas foram obtidos no aparelho CG/EM Varian Saturn 2000 com ionização de impacto de elétrons.

\section{Material botânico}

O material vegetal foi coletado no Morro de São Sebastião, Ouro Preto, Minas Gerais, Brasil, e autenticado através de comparação com exsicata (№ 6732) depositada no Herbário OUPR-UFOP, curadora do herbário Dra Maria Cristina Trivelato Messias, Instituto de Ciências Exatas e Biológicas da Universidade Federal de Ouro Preto, Ouro Preto-MG, Brasil. 


\section{Extração e Isolamento dos constituintes químicos}

O caule seco e triturado (500,0 g) de Laseguea erecta foi submetido a extração através de maceração com hexano e em seguida com metanol até exaustão. Os solventes das soluções dos extratos foram retirados através de destilação em evaporador rotativo, obtendose, após total evaporação do solvente, os resíduos dos extratos LECH (30,8 g) e LECM (11,6 g). A análise dos espectros IV e RMN ${ }^{1} \mathrm{H}$ de LECH revelou ser constituído apenas de hidrocarbonetos alifáticos saturados. O extrato LECM foi submetido a partição com solventes orgânicos. Para isso foi dissolvido em metanol:água (9:1), extraído sucessivamente com hexano, diclorometano e acetato de etila, fornecendo, respectivamente, as frações LECMH (197,0 mg), LECMD (2,0 g), LECMA (270,0 mg) e $\operatorname{LECMM~(3,0~g).~}$

O resíduo LECMA revelou-se pouco solúvel em clorofórmio. Acetilação com anidrido acético na presença de piridina (1:1) forneceu $236,0 \mathrm{mg}$ de produto acetilado. Este produto foi submetido a filtração em coluna de sílica gel, recolhendo-se 6 frações. As frações reunidas 1-3 $(30,0 \mathrm{mg})$ foram analisadas através de espectros de IV e RMN ${ }^{1} \mathrm{H}$ e foi identificado como derivado acetato de lupelila (1a); as demais frações revelaram-se constituídas de mistura de álcoois alifáticos. O resíduo obtido com diclorometano $(2,0 \mathrm{~g})$ foi submetido a fracionamento em coluna de sílica gel, recolhendo-se 24 frações que foram analisadas com CCDA e reunidas em grupos de acordo com a semelhança na revelação com vapor de iodo. A fração 1-4 foi submetida a fracionamento em coluna de Sephadex LH-20, obtendo-se 12 frações; nas frações LECMD-1-4/3 e LECMD-1-4/6 foram identificados 1 $(8,0 \mathrm{mg})$ e $2(6,0 \mathrm{mg})$, respectivamente. E a fração 510 (das 24 frações da primeira coluna) foi submetida também a uma coluna de Sephadex LH-20, obtendo-se nas primeiras frações a substância $3(3,0 \mathrm{mg})$. A análise dos espectros de IV e RMN ${ }^{1} \mathrm{H}$ das últimas frações desta coluna permitiu reconhecer a presença de mistura de $\mathbf{3}$ com outros álcoois alifáticos. O tratamento de $\mathbf{1}$ com anidrido acético/piridina (1:1) forneceu 1a , que serviu para confirmar a estrutura proposta para $\mathbf{1}$.

3-(3-O-metilrhamnosil)-digitoxigenina ou $\alpha-L$ tevetosil-digitoxigenina (2): $\mathrm{RMN}{ }^{1} \mathrm{H}\left(\mathrm{CDCl}_{3}, 400 \mathrm{MHz}\right)$ : $\delta_{\mathrm{H}}$ (multiplicidade, $J$ em Hz, atribuição): 1,25-1,26 (m, H-1 e H-2), 4,05 (sl, H-3), 1,49 (sl, H-4), 1,66 (m, H-5), 1,87 (m, H-6), 1,64 e 1,20 (H-7), 1,54 (H-8), 1,58 (H-9), 1,97 e 1,43 (2H-11), 1,39 (H-12), 2,08 e 1,68 (2H-15), 1,70 e 1,54 (2H-16), 2,77 (dd, $J=9,2$ e 4,5, H-17), 0,87 (s, $3 \mathrm{H}-18), 0,94$ (3H-19), 4,80 e 4,98 (dd, $J=18,2$ e $1,6 \mathrm{~Hz}$, $2 \mathrm{H}-21$ ), 5,88 (s, H-22), 4,25 (d, J=7,8 Hz, H-1'), 3,65 (t, $J=9,0 \mathrm{~Hz}, \mathrm{H}-2$ '), 3,22 (dd, $J=9,2$ e 3,4 Hz, H-3'), 3,85 (dl, $J=3,0 \mathrm{~Hz}, \mathrm{H}-4$ ') $, 3,57$ (q, $J=6,4 \mathrm{~Hz}, \mathrm{H}-5$ ') $, 1,35$ (d, $J=6,4$ $\mathrm{Hz}, \mathrm{H}-6$ '), 3,53 (s, MeO-3').

\section{RESULTADOS E DISCUSSÃO}

O lupeol 1 foi identificado através da análise do espectro de IV e $\mathrm{RMN}{ }^{1} \mathrm{H}$ e ${ }^{13} \mathrm{C}$ do derivado acetilado (1a) envolvendo a comparação com os dados do derivado isolado de Parahancornia amapa (Sobrinho et al., 1991 e Carvalho et al., 2001b).

O espectro no IV da substância 2 apresentou bandas de absorção de hidroxila $\left(v_{\text {máx }} 3468 \mathrm{~cm}^{-1}\right)$, grupamentos $\mathrm{CH}_{2}$ e $\mathrm{CH}_{3}\left(v_{\text {máx }} 2934\right.$ e $\left.2861 \mathrm{~cm}^{-1}\right)$, carbonila de $\gamma$-lactona $\alpha, \beta$-conjugada $\left(v_{\text {máx }} 1745 \mathrm{~cm}^{-1}\right), \quad \mathrm{C}=\mathrm{C}$ conjugada $\left(v_{\text {máx }} 1625 \mathrm{~cm}^{-1}\right)$ e C-O $\left(v_{\text {máx }} 1263-1071 \mathrm{~cm}^{-1}\right)$. $\mathrm{O}$ espectro de RMN ${ }^{1} \mathrm{H}$ desta substância apresentou sinais de hidrogênio olefínico em $\delta_{\mathrm{H}} 5,88(\mathrm{~s}, 1 \mathrm{H})$, dois duplo dubletos em $\delta_{\mathrm{H}} 4,80$ e 4,98 ( $J=18,2$ e $1,6 \mathrm{~Hz}, 1 \mathrm{H}$ cada $)$ de um grupo metileno oxigenado, um dubleto em $\delta_{\mathrm{H}} 4,25$ $(J=7,8 \mathrm{~Hz})$ e um singleto largo em $\delta_{\mathrm{H}} 4,05$. Um grupo de sinais adicionais foi usado para caracterizar a presença de uma unidade de carboidrato. A análise comparativa dos espectros de RMN ${ }^{13} \mathrm{C}$-HBBD e RMN ${ }^{13} \mathrm{C}$-DEPT (100 $\mathrm{MHz}$ ) permitiu identificar o número de $\mathrm{CH}_{3}, \mathrm{CH}_{2}, \mathrm{CH}$ e C, e o espectro 2D HMQC foi usado para a correlação heteronuclear dos valores de deslocamentos químicos dos carbonos hidrogenados correspondentes. Além do sistema $\gamma$-lactonônico $\alpha, \beta$-insaturado, representado pelos sinais correspondentes aos átomos de carbono em $\delta_{\mathrm{C}} 174,5$ (C-23 ou C-20), 174,4 (C-20 ou C-23) e 117,7 $\left(\mathrm{CH}-21\right.$, ligado ao $\left.\mathrm{H} \operatorname{com} \delta_{\mathrm{H}} 5,88\right)$ e $73,4\left(\mathrm{CH}_{2}\right.$, ligado aos hidrogênios com $\delta_{\mathrm{H}} 4,80$ e 4,98), os espectros de RMN ${ }^{13} \mathrm{C}$ (HBBD) e HMQC apresentaram sinais que permitiu identificar um $\mathrm{CH}$ em 73,9 (ligado ao $\mathrm{H}$ com $\delta_{\mathrm{H}} 4,05$ ) e os sinais da 3- $O$-metilrhamnose: $\delta_{\mathrm{CH}} 101,2\left(\mathrm{CH}-1^{\prime}\right)$, 70,8 (CH-4'), 82,8 (CH-3'), 68,2 (CH-2'), 70,4 (CH-5'), e $\delta_{\mathrm{CH} 3} 16,5\left(\mathrm{H}_{3} \mathrm{C}-6\right.$ ') ligados aos respectivos hidrogênios em $\delta_{\mathrm{H}} 4,25$ (d, $J=7,8 \mathrm{~Hz}, \mathrm{H}-1$ ') $, 3,65$ (t, $J=9,0 \mathrm{~Hz}, \mathrm{H}-4$ '), 3,22 (dd, $J=9,0$ e 3,4 Hz, H-3'), 3,85 (dl, $J=3,0$ Hz, H-2'), 3,57 (m, H-5'), 1,35 (d, $J=6,4$ Hz, 3H-6')]. A ausência de sinal de acoplamento a uma ligação $\left({ }^{1} J_{\mathrm{CH}}\right)$ permitiu identificar um carbono oxigenado quaternário com $\delta_{\mathrm{C}}$ 85,6 . Além destes sinais, foi ainda possível identificar as absorções adicionais de dois grupos metílicos $\left[\left(\mathrm{CH}_{3}\right)_{2}\right]$, nove metilênicos $\left[\left(\mathrm{CH}_{2}\right)_{9}\right]$, quatro metínicos $\left[(\mathrm{CH})_{4}\right]$, e confirmar a presença de dois carbonos quaternários $\left[(\mathrm{C})_{2}\right]$ e um grupo metoxílico (MeO-3'). Todos os dados espectrais acima interpretados, incluindo-se a comparação com valores da literatura (Abe et al., 1992), permitiram propor a estrutura do heterosídeo esterólico 2 [3(3-Ometilrhamnosil)-digitoxigenina) e a completa atribuição dos deslocamentos químicos dos átomos de hidrogênio e carbono. $\mathrm{O}$ espectro de massas apresentou picos em $m / z 357\left(\left[\mathrm{M}-\mathrm{C}_{7} \mathrm{H}_{13} \mathrm{O}_{5}\right]^{+}, \mathbf{2 a}, 40 \%\right), 339\left(\left[357-\mathrm{H}_{2} \mathrm{O}\right]^{+}\right.$, 2b, $45 \%), 177\left(\left[\mathrm{C}_{7} \mathrm{H}_{13} \mathrm{O}_{5}\right]^{+}, \mathbf{2 c}, 15 \%\right)$, que contribuíram para a confirmação da estrutura proposta. O pico em $\mathrm{m} / \mathrm{z}$ 177 (2c) foi também utilizado para a caracterização da presença da unidade $O$-metilrhamnose.

$\mathrm{O}$ espectro no IV de $\mathbf{3}$ apresentou bandas em $v_{\text {máx }} 3442(\mathrm{OH}), 1270(\mathrm{C}-\mathrm{O}), 1721(\mathrm{C}=\mathrm{O})$ e 1642,1517 e 918 (aromático e ligação dupla olefínica) $\mathrm{cm}^{-1}$. O espectro de $\mathrm{RMN}{ }^{13} \mathrm{C}$ (HBBD) apresentou quatro sinais 


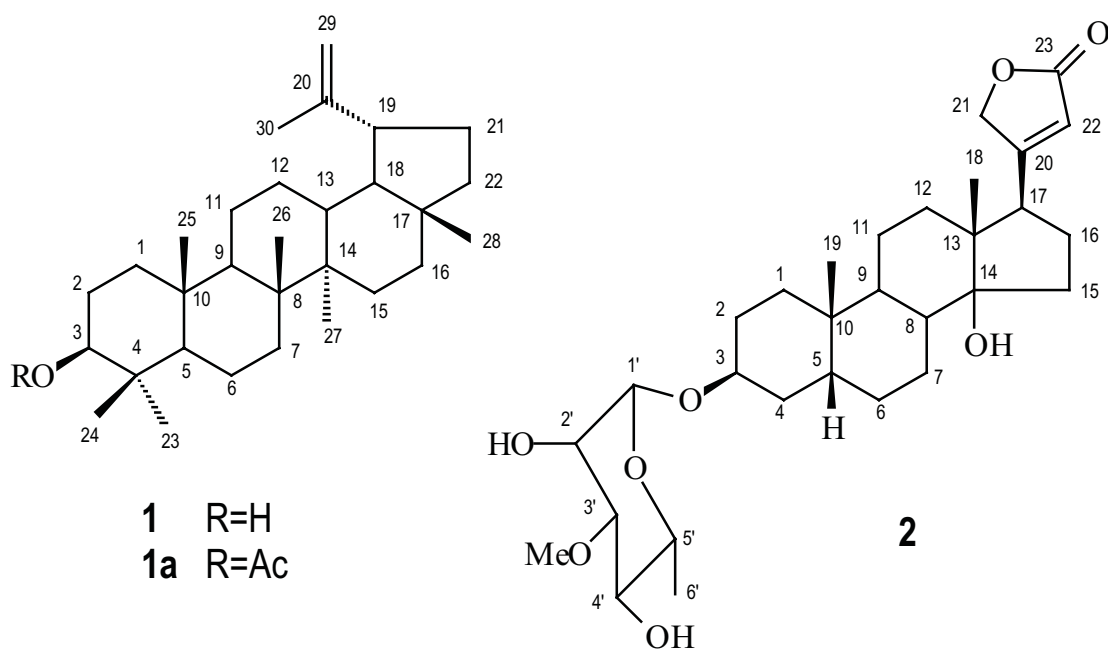<smiles>[R]Oc1cc2oc(=O)ccc2cc1OC</smiles>

$3 \mathrm{R}=\mathrm{H}$ 3a $\mathrm{R}=\mathrm{Me}$<smiles>COc1cc([C@H]2OC[C@H]3[C@H](c4ccc(O)c(OC)c4)OC[C@H]23)ccc1O</smiles>

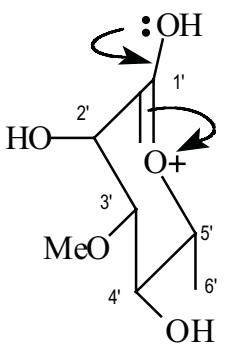<smiles>[C-]#Cc1ccc(O)c(OC)c1</smiles>

2c $\mathrm{m} / \mathrm{z} 177$<smiles>CC12CCC3C(CC[C@@H]4C[CH+]CC[C@]34C)C1(O)CC[C@@H]2C1=CC(=O)OC1</smiles>

2a $m / z 357(40 \%)$<smiles>CC12CCC3C4CCC3(C)[C@@H]3CCC[C@](C)(C3)C4=C1CCC2[C@@H]1COC(=O)C1</smiles>

2b $m / z 339(45 \%)$ intensos de carbonos metínicos em $\delta_{\mathrm{CH}} 143,8,113,3$, 107,4 e 103,2 , um sinal em $\delta_{\text {OMe }} 56,4$ representando uma metoxila, além de absorções em $\delta_{\mathrm{C}} 161.5,149,7,150,0$, $144,0,111,0$ de carbonos quaternários. O espectro de RMN ${ }^{1} \mathrm{H}\left(200 \mathrm{MHz}, \mathrm{CD}_{3} \mathrm{OD}\right)$ apresentou dois singletos em $\delta_{\mathrm{H}} 7,09(\mathrm{H}-8)$ e $6,75(\mathrm{H}-5)$, e dois dubletos $(J=9,4$ $\mathrm{Hz})$ em $\delta_{\mathrm{H}} 6,20(\mathrm{H}-3)$ e $7,80(\mathrm{H}-4)$. Estes dados permitiu caracterizar um esqueleto de uma cumarina (3) contendo dois substituintes no anel aromático, um $\mathrm{OH}(\mathrm{sl}, 8,5$ ppm) e uma metoxila $\left(\delta_{\mathrm{H}} 3,89, \mathrm{~s}, 3 \mathrm{H}\right)$ nas posições $6 \mathrm{e}$ 7. Esta estrutura revelou-se compatível com o valor do pico $(\mathrm{m} / \mathrm{z} 192,100 \%)$ correspondente ao íon molecular $\left([\mathrm{M}]^{+}\right)$e os picos adicionais em $\mathrm{m} / z 177\left([\mathrm{M}-\mathrm{Me}]^{+}, 60\right.$ $\%), 164\left([\mathrm{M}-\mathrm{CO}]^{+}, 40 \%\right), 149\left([\mathrm{M}-\mathrm{Me}-\mathrm{CO}]^{+} \mathrm{e} / \mathrm{ou}[\mathrm{M}\right.$
- $\left.\mathrm{CO}-\mathrm{Me}]^{+}, 50 \%\right)$ e $121\left(\left([\mathrm{~m} / z 149-\mathrm{CO}]^{+}, 30 \%\right)\right.$. Os deslocamentos químicos de hidrogênio e carbono-13 de 3 foram comparados com os valores corrigidos através de técnicas especiais de RMN e registrados na literatura (Vasconcelos et al., 1998). Esta comparação confirmou a proposta da escopoletina para 3. O tratamento de $\mathbf{3}$ com diazomentano forneceu $\mathbf{3 a}$ cujo espectro de RMN ${ }^{1} \mathrm{H}\left(200 \mathrm{MHz}, \mathrm{D}_{3} \mathrm{CCOCD}_{3}\right)$ apresentou sinais em $\delta_{\mathrm{H}} 7,87$ (d, J=9,6 Hz, H-4), 7,17 (s, H-5), 6,95 (s, H-8), 6,21 (d, $J=9,6, \mathrm{H}-3), 3,94$ (s, MeO) e 3,86 (s, MeO). Os espectros obtidos com NOEDIFF (200 MHz) com irradiação em: a) em $\delta_{\mathrm{H}} 3,85(\mathrm{MeO}-6)$ revelou NOE em $\delta_{\mathrm{H}} 7,17$ (H-5); b) em $\delta_{\mathrm{H}} 3,93(\mathrm{MeO}-7)$ apresentou NOE em $\delta_{\mathrm{H}} 6,95(\mathrm{H}-$ 8); c) em $\delta_{\mathrm{H}} 7,17(\mathrm{H}-5)$ gerou NOE em $\delta_{\mathrm{H}} 7,87(\mathrm{H}-4)$. 
Esta análise permitiu fazer atribuição inequívoca dos deslocamentos químicos das metoxilas $\delta_{\mathrm{H}} 3,93(\mathrm{MeO}-$ 7), 3,85 (MeO-6) e dos hidrogênios H-5 e H-8 em 7,17, e 6,95 , respectivamente. Sendo, assim, a estrutura de $\mathbf{3}$ foi definida como 7-hidroxi-6-metoxi-2H-1-benzopiran2-ona, isolada anteriormente de Guarea rhophalocarpa (Camacho et al., 2001) e de Artemisia campestris subsp. maritima (Vasconcelos et al., 1998) conhecida como escopoletina.

A substância 4 foi identificada através da análise dos espectros de hidrogênio $(400 \mathrm{MHz})$ incluindo NOESY, espectro de massas e comparação com dados da literatura (Cuenca, Catalan, 1991). A presença de sistema aromático 1,2,4-tri-substituído foi reconhecida pelos sinais em $\delta_{\mathrm{H}} 6,98$ (d, $J=2,0, \mathrm{~Hz}, \mathrm{H}-2 / \mathrm{H}-2$ ') $, 6,78$ (d, $J=8,0$ $\mathrm{Hz}, \mathrm{H}-5 / \mathrm{H}-5$ ') e 6,82 (dd, $J=8,0$ e $2,0 \mathrm{~Hz}, \mathrm{H}-6 / \mathrm{H}-6$ ') no espectro de $\mathrm{RMN}{ }^{1} \mathrm{H}$. Este espectro apresentou também um sinal de grupo metoxílico em $\delta_{\mathrm{H}} 3,84$. Os demais sinais em $\delta_{\mathrm{H}} 4,66$ (d, $J=4,4 \mathrm{~Hz}, \mathrm{H}-7 / \mathrm{H}^{-7}$ '), 4,19 (dd, $J=9,0$ e 7,0 Hz, H-9a/H-9'a), 3,80 (dd, $J=9,0$ e 4,4 Hz, H9b/H-9'b) e 3,08 (m, H-8/H-8'). Os picos em m/z 151 (4a, $100 \%), 137$ (4b, $50 \%)$ e 123 (4c, $19 \%$ ) no espectro de massas permitiram sugerir a presença de anel aromático sustentando um grupo hidroxila e uma metoxila. O pico $\mathrm{M}^{+}$. em $m / z 358$ (29\%) revelou-se compatível com a fórmula molecular $\mathrm{C}_{18} \mathrm{H}_{14} \mathrm{O}_{2}(\mathrm{OH})_{2}\left(\mathrm{OCH}_{3}\right)_{2}$, em acordo com lignana furofurânica contendo dois grupos guaiacila (4). A posição da metoxila em 3',3 foi deduzida pelo sinal de NOE revelado pelo espectro de NOESY, revelando interação entre a metoxila (MeO-3'/MeO-3) e os hidrogênios H-2/H-2'. A comparação desses dados com valores da literatura para lignanas furofurânicas permitiu definir a estrutura de 4 como o pinoresinol (Cuenca, Catalan, 1991)

\section{AGRADECIMENTOS}

Os autores agradecem ao CNPq, CAPES e a FAPERJ pelas bolsas e suporte financeiro concedidos.

\section{REFERÊNCIAS}

Abe R, Yamauchi T, Nohara T 1992. C-Nor-D-homo-cardenolide glycosides from Thevetia neriifolia. Phytochemistry 31: 251-254.

Barbosa-Fiho JM, Medeiros KCP, Diniz MFFM, Batista LM, Athayde-filho PF, Silva MS, Cunha EVL, Almeida JRGS, Quintans-Junior LJ 2006. Natural products inhibitors of the enzime acetylcholinesterase. Rev Bras Farmacogn 16: 258-285.

Barreto AS, Carvalho MG, Nery IA, Gonzaga L, Kaplan MAC 1998. Chemical constituents from Himatanthus articulata. J Braz Chem Soc 9: 430-434.

Camacho MR, Phillipson JD, Croft SL, Kirby GC, Warhurst DC, Solis PN 2001. Terpenoids from Guarea rhophalocarpa. Phytochemistry 56: 203-210.

Carvalhaes SF, Costa DL, Mazzei JL, Taddei LEM, d'Avila LA 2002. Alternative extraction of alkaloid anticarcinogens from Brazilian "vinca rósea" using long exchange chromatography. Rev Bras Framacogn 12 (supl.): 83-34

Carvalho MG, Cranchi DC, Kingston DGI, Werle AA 2001a. Proposed active constituents of Dipladenia martiana. Phytother Res 15: 715-717.

Carvalho MG, Velloso CRX, Braz-Filho R. Costa WF 2001b. Acyl-lupeol esters from Parahancornia amapa (Apocynaceae). J Braz Chem Soc 12: 556-559.

Cuenca MDR, Catalan CAN 1991. Monoterpenes and lignans from Mikania saltensis. J Nat Prod 54: 1162-1164.

Falcão HS, Lima IO, Santos VL, Dantas HF, Diniz MFFM, Barbosa-Filho JM, Batista LM 2005. Review of the plants with anti-inflammatory activity studied in Brazil. Rev Bras Farmacogn 15: 381-391.

Morais SMM, Dantas JDP, Silva ARA, Magalhães EF 2005. Plantas medicinais usadas pelos índios Tapebas do Ceará. Rev Bras Farmacogn 15: 169-177.

Nishibe S 1989. Structure elucidation and biological activities of phenylpropanoids, coumarins and lignans from medicinal plants. In: Studies in Natural Products Chemistry, V.5, NY, Atta-ur-Rahman ed. Elsevier, p. 511 e 544.

Sobrinho DC, Hauptli MB, Appolinário EV, Kollenz CLM, Carvalho MG, Braz-Filho R 1991. Triterpenoids isolated from Parahancornia amapa, J Braz Chem Soc 2: 15-20.

Sousa MP, Matos MEO, Matos FJA, Machado MIL, Craveiro AA2004. Constituintes quimicos ativos e propriedades biológicas de plantas medicinais Brasileiras. Fortaleza-CE, 2 a Ed., Editora da UFC, p. 216.

Vanderlei MF, Silva MS, Gottlieb HE, Braz-Filho R 1991. Iridoids and triterpenes from Himatanhthus phagadaenica, The complete assignment of the ${ }^{1} \mathrm{H}$ and ${ }^{13} \mathrm{C}$ NMR spectra of two Iridoids glycosides. $J$ Braz Chem Soc 2: 51-55,

Vasconcelos JMJ, Silva MAS, Cavaleiro JAS 1998. Chromones and flavanones from Artemisia campestris Subsp. Maritima. Phytochemistry 49: 1421-1424. 